Technical note

\title{
Dosimetry in magnetic fields with dedicated MR-compatible ionization chambers
}

\author{
Bhargesh K. Shukla $^{\text {a,b, *, Claudia Katharina Spindeldreier }}{ }^{\text {a,b,c }}$, Oliver Schrenk ${ }^{\text {a,b,c,d, }}$ \\ Anna C. Bakenecker ${ }^{a, b}$, Sebastian Klüter ${ }^{\text {b, }}$, Iwan Kawrakow ${ }^{e}$, Armin Runz ${ }^{\text {a,b }}$, Lucas Burigo ${ }^{\text {a,b }}$, \\ Christian P. Karger ${ }^{\mathrm{a}, \mathrm{b}}$, Steffen Greilich ${ }^{\mathrm{a}, \mathrm{b}}$, Asja Pfaffenberger ${ }^{\mathrm{a}, \mathrm{b}}$ \\ ${ }^{a}$ Medical Physics in Radiation Oncology, German Cancer Research Center (DKFZ), Heidelberg, Germany \\ ${ }^{\mathrm{b}}$ National Center for Radiation Research in Oncology (NCRO), Heidelberg Institute for Radiooncology (HIRO), Heidelberg, Germany \\ ${ }^{\mathrm{c}}$ Department of Radiation Oncology, University Hospital Heidelberg, Heidelberg, Germany \\ ${ }^{\mathrm{d}}$ Medical Faculty, University of Heidelberg, Heidelberg, Germany \\ ${ }^{\mathrm{e}}$ Viewray Inc., Thermo Fischer Way, Oakwood village, OH, USA
}

\section{A R T I C L E I N F O}

\section{Keywords:}

MR-guided radiotherapy

MR-linac

Dosimetry in magnetic fields

Monte-Carlo simulation

EGSnrc

MR-compatible ionization chambers

Polarity effect

Recombination effect

\begin{abstract}
A B S T R A C T
MR-integrated radiotherapy requires suitable dosimetry detectors to be used in magnetic fields. This study investigates the feasibility of using dedicated MR-compatible ionization chambers at MR-integrated radiotherapy devices. MR-compatible ionization chambers (Exradin A19MR, A1SLMR, A26MR, A28MR) were precisely modeled and their relative response in a $6 \mathrm{MV}$ treatment beam in the presence of a magnetic field was simulated using EGSnrc. Monte Carlo simulations were carried out with the magnetic field in three orientations: the magnetic field aligned perpendicular to the chamber and beam axis (transverse orientation), the magnetic field parallel to the chamber as well as parallel to the beam axis. Monte Carlo simulation results were validated with measurements using an electromagnet with magnetic field strength upto $1.1 \mathrm{~T}$ with the chambers in transverse orientation. The measurements and simulation results were in good agreement, except for the A26MR ionization chamber in transverse orientation. The maximum increase in response of the ionization chambers observed was 8.6\% for the transverse orientation. No appreciable change in chamber response due to the magnetic field was observed for the magnetic field parallel to the ionization chamber and parallel to the photon beam.

Polarity and recombination correction factor were experimentally investigated in the transverse orientation. The polarity effect and recombination effect were not altered by a magnetic field.

This study further investigates the response of the ionization chambers as a function of the chambers' rotation around their longitudinal axis. A variation in response was observed when the chamber was not rotationally symmetric, which was independent of the magnetic field.
\end{abstract}

\section{Introduction}

Adaptive treatment planning with a new modality that integrates magnetic resonance imaging and a radiation therapy unit (MR-Linac) is a key development in radiation oncology. The excellent soft-tissue visualization in MRI improves targeted dose delivery with the aim to reduce complications or improve treatment outcome. MR-guidedRadiotherapy (MRgRT) can provide real-time imaging at the time of treatment. A few research groups have advanced with various designs of MR-Linac; the Aurora RT Linac-MR by Magnet $\tau x$ [1] (Canada) and the Australian MRI-Linac [2] use a magnetic field parallel to the photon beam, while the ViewRay MRIdian linac ${ }^{\mathrm{fi}}[3,4]$ and the Elekta Unity with Philips MR technology [5] employ a magnetic field perpendicular to the photon beam.

For dosimetric measurements in magnetic fields, it has been shown that the detector response can be affected by the orientation of the magnetic field relative to chamber orientation and beam direction [6-12]. The trajectory of the secondary electrons in the presence of magnetic field are affected by the Lorentz force, with the force depending on the energy of the electron and the magnetic field strength. In ionization chambers where the sensitive volume is filled with air, electrons will follow curved trajectories [6]. The response of chambers is known to depend on the chamber type, the magnetic field strength and the orientation between the chamber axis, beam and magnetic field

\footnotetext{
* Corresponding author.
} 
Table 1

Specifications of the ionization chambers as provided by the manufacturer.

\begin{tabular}{|c|c|c|c|c|}
\hline & A1SLMR & A28MR & A26MR & A19MR \\
\hline & $\begin{array}{l}\text { Slimline } \\
\text { miniature }\end{array}$ & Scanning & Micropoint & $\begin{array}{l}\text { Farmer- } \\
\text { type }\end{array}$ \\
\hline Collecting volume & $0.053 \mathrm{cc}$ & 0.125 cc & $0.015 \mathrm{cc}$ & $0.62 \mathrm{cc}$ \\
\hline $\begin{array}{l}\text { Outside diameter of } \\
\text { shell collecting } \\
\text { volume }\end{array}$ & $6.35 \mathrm{~mm}$ & $8.0 \mathrm{~mm}$ & $4.3 \mathrm{~mm}$ & $7.1 \mathrm{~mm}$ \\
\hline $\begin{array}{l}\text { Inside diameter of } \\
\text { shell collecting } \\
\text { volume }\end{array}$ & $4.0 \mathrm{~mm}$ & $5.8 \mathrm{~mm}$ & $3.3 \mathrm{~mm}$ & $6.1 \mathrm{~mm}$ \\
\hline $\begin{array}{l}\text { Centroid of collecting } \\
\text { volume (From tip to } \\
\text { chamber) }\end{array}$ & $4.1 \mathrm{~mm}$ & $4.47 \mathrm{~mm}$ & $1.98 \mathrm{~mm}$ & $13.0 \mathrm{~mm}$ \\
\hline Shell wall thickness & $1.1 \mathrm{~mm}$ & $1.1 \mathrm{~mm}$ & $0.5 \mathrm{~mm}$ & $0.5 \mathrm{~mm}$ \\
\hline Collector diameter & $1.0 \mathrm{~mm}$ & $1.0 \mathrm{~mm}$ & $0.75 \mathrm{~mm}$ & $1.0 \mathrm{~mm}$ \\
\hline Collector length & $4.4 \mathrm{~mm}$ & $6.4 \mathrm{~mm}$ & 1.78 & $21.6 \mathrm{~mm}$ \\
\hline $\begin{array}{l}\text { Shell/Entry window, } \\
\text { collector and guard } \\
\text { material }\end{array}$ & C552 & C552 & C552 & C552 \\
\hline
\end{tabular}

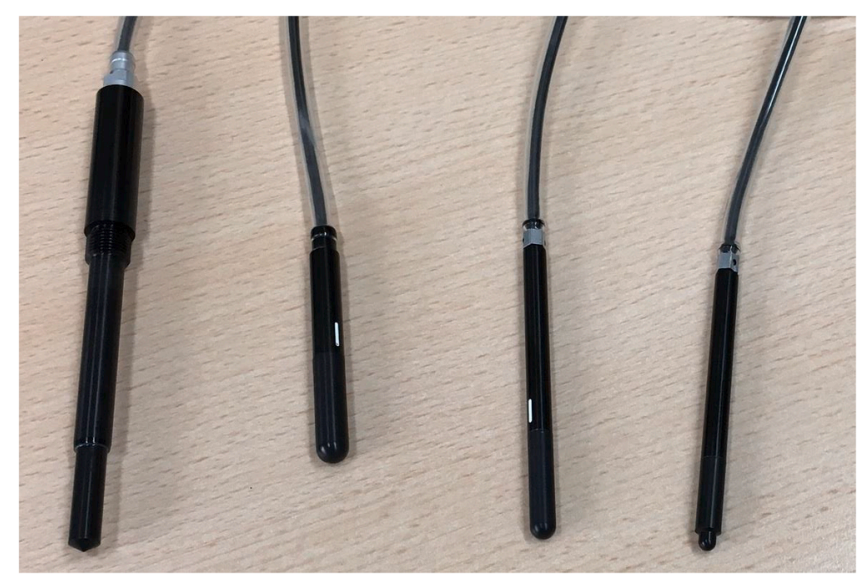

Fig. 1. The MR-compatible ionization chambers used; namely (left to right) A19MR, A28MR, A1SLMR and A26MR.

$[9,10]$. Recently, the available data were summarized for a range of ionization chambers [13], but for some relevant chambers data are still lacking.
This study investigates the response of recently developed MRcompatible ionization chambers with different cavity sizes in a magnetic field. These chambers are dedicated for use at MR-linacs and their response in presence of magnetic fields is therefore required for accurate dose measurements. The ionization chamber response was studied as a function of the magnetic field strength and its orientation relative to the photon beam. The measurements were performed in an electromagnet positioned at a clinical linac. Also, measurements were compared with Monte-Carlo radiation transport simulations. In addition, the impact of the magnetic field on the polarity and recombination correction as well as on the response to axial rotations of the chamber were studied.

\section{Materials and methods}

\subsection{Ionization chambers}

The response of four different Exradin MR-compatible ionization chambers (A19MR, A28MR, A26MR and A1SLMR) from Standard Imaging Inc. (Wisconsin, USA) [14] was studied. Table 1 shows the geometry specifications.

\subsection{Relative response for different magnetic field strengths and orientations}

\subsubsection{Experimental setup}

Measurements were performed using a medical linear accelerator with a 6 MV beam with flattening filter (Artiste linac, Siemens Medical Solutions Inc., PA, USA) and the four different ionization chambers (Fig. 1) placed in a water phantom between the pole shoes of an electromagnet (AGEM 5520, Schwarzbeck Mess-Elektronik OHG, Germany). A 3D-printed MR-compatible water phantom with a holder for the ionization chambers was positioned at a standard $100 \mathrm{~cm}$ source-tosurface distance (SSD) to the water surface with the field size of $10 \times$ $3 \mathrm{~cm}$. The ionization chamber was positioned at $10 \mathrm{~cm}$ water-equivalent depth between the magnet pole shoes. The tank was printed by a 3D printer using VeroClear RGD 810 as printing material (density of 1.18 $1.19 \mathrm{~g} / \mathrm{cm}^{3}$ ). The wall thickness on the entrance side was $0.5 \mathrm{~cm}$ and the geometrical length of the water tank was $14.8 \mathrm{~cm}$ with a surface to measurement point distance of $9.91 \mathrm{~cm}$ (10 cm water-equivalent depth). Fig. 2 shows a schematic drawing of the water phantom. The tank was designed in a way that the deepening on the lateral sides match the shape of the pole shoes for a pole shoe distance of $3.5 \mathrm{~cm}$. Measurements with variable field size asserted that this distance was sufficient to
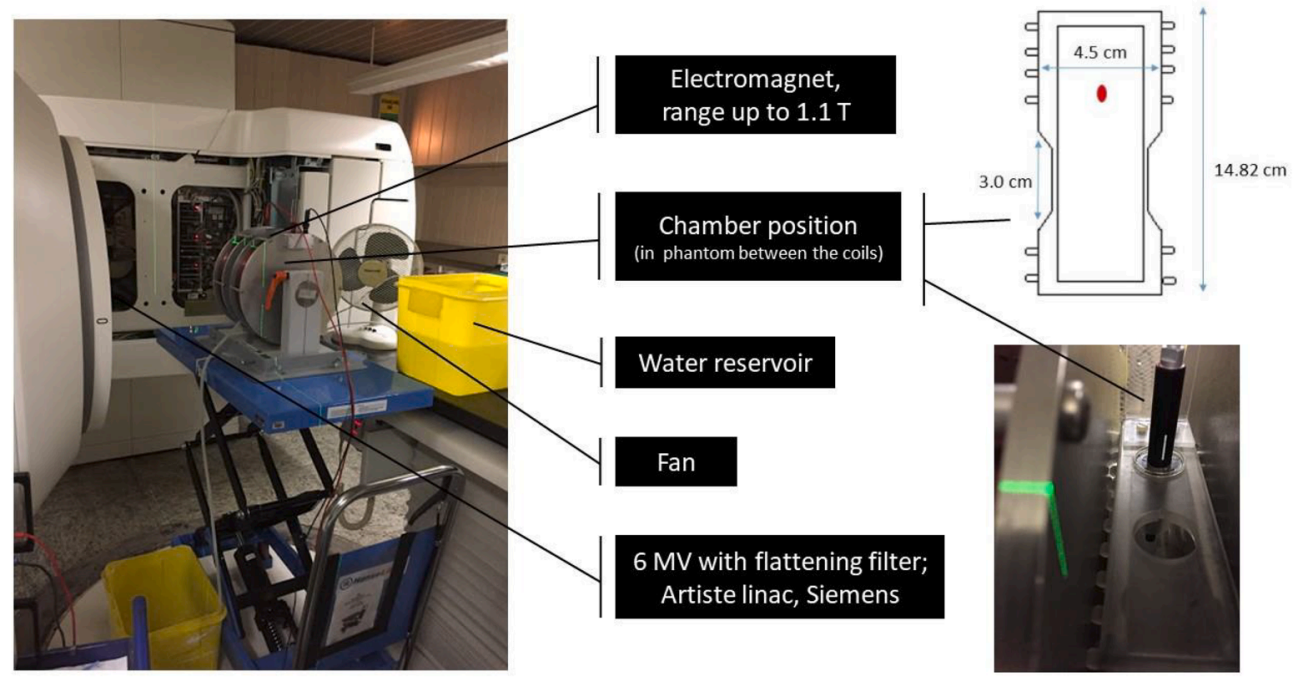

Fig. 2. Photograph of the experimental setup together with schematic drawing of the water tank (the red dot indicates the $10 \mathrm{~cm}$ water equivalent depth for the position of the ionization chambers). (For interpretation of the references to colour in this figure legend, the reader is referred to the web version of this article.) 


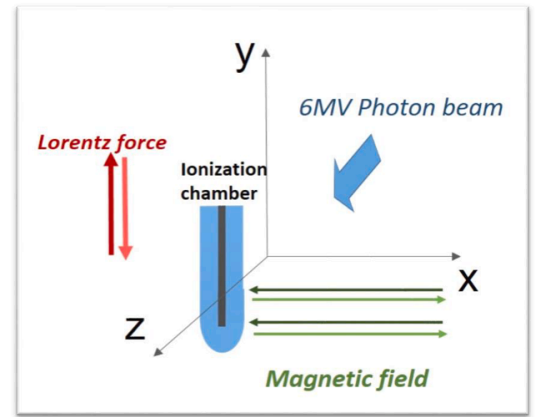

(a)

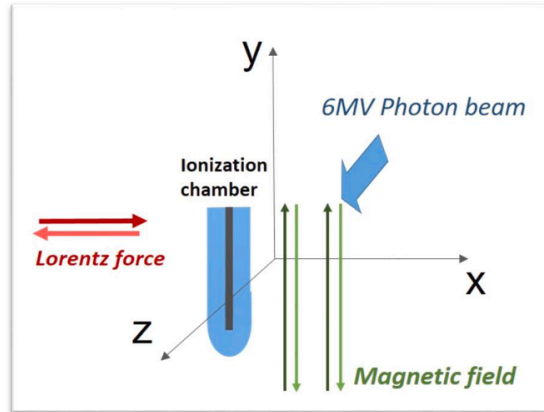

(b)

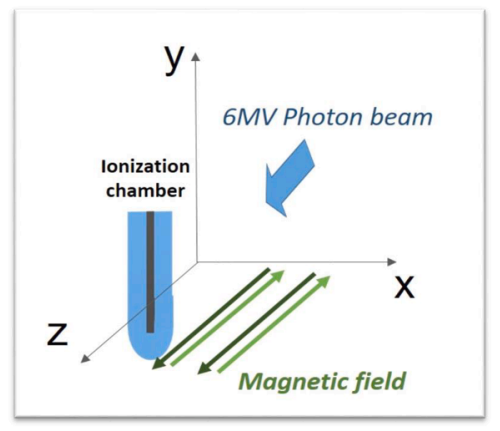

(c)

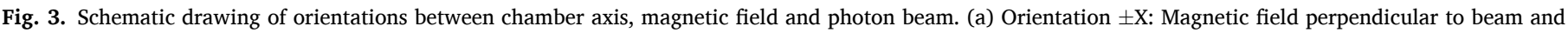

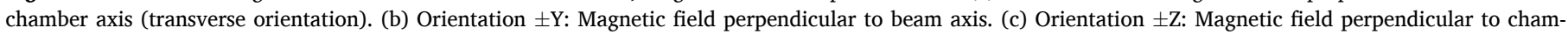
ber axis.

exclude significant scatter contributions at the position of the chamber.

As the electromagnet tends to heat up during operation, the contact area between the tank and pole shoes was minimized by small nubs. In addition, the water in the water phantom was continuously exchanged with water from a reservoir by means of a circulation pump and a fan was placed next to the magnet to remove the heat created by the magnet coils. The experimental setup is shown in Fig. 2.

Due to the geometry of the setup, the experimental measurement was only feasible for the magnetic field perpendicular to the chamber axis and for the beam axis perpendicular to the magnetic field and the chamber axis (transverse orientation) (Fig. 3a). The ionization chamber was exposed by a magnetic field ranging up to $1.1 \mathrm{~T}$ in intervals of $0.1 \mathrm{~T}$. The bias voltage was set to $+300 \mathrm{~V}$. The chamber response was measured three times for each magnetic field strength. In each of the three sets of measurements, the order of field strength was randomized to reduce hysteresis effects. The measurements were repeated by reversing the polarity of the electromagnet. The correction factor for temperature and pressure, $k_{T, P}$, was determined for each set of measurements and was found to be constant within $0.96 \%$.

\subsection{Simulation setup}

The Monte-Carlo code system EGSnrc [15] with charged particle transport under electromagnetic fields macro was used for the simulations of the ionization chambers. The dose deposited in the cavity of the ionization chamber was calculated using the user code egs chamber $[16,17]$. Also, to improve the efficiency of ionization chamber simulation, variance reduction techniques like cross section enhancement (XCSE) and range based Russian Roulette are implemented in the user code egs chamber. The Exradin ionization chambers were modeled in egs ++ using the technical data provided by the manufacturer and verified with CT images (Inveon, Siemens, Erlangen, Germany).

The simulation modeled the experimental setup employing a photon beam with a generic energy spectrum of a $6 \mathrm{MV}$ [18]. The simulation was carried out for three different orientations (Fig. 3): The orientations of the beam and the chambers were fixed while the magnetic field was selected along the $\pm \mathrm{X}, \pm \mathrm{Y}$ and $\pm \mathrm{Z}$ direction, respectively. The experimental measurement in transverse $( \pm X)$ orientation was used to validate the simulation and the other two orientations were then simulated using these settings (see [12] for details).

It is well-known that the charge collection in ionization chambers does not only depend on the geometry of the air cavity but also on the details of the electric field and due to the details of the construction there may be dead volumes that do not contribute to the charge collection. By simulating the electric field in the ion chamber, it has been shown that the dead volume is located close to the stem and the guard electrode [19]. For the A26MR, we modeled the dead volume adjacent to the guard electrode as cylinders with the radius of the geometrical volume of the ionization chamber and adjusted the height to bring the simulation results in accordance with experimental results. For the other ion chambers, this adjustment was not necessary. This approach to consider the dead volume has also been used by Spindeldreier et al. (2017) for other chambers [12].

The total energy deposition inside the sensitive volume of the ionization chamber was scored as a function of the magnetic field strength. The relative response of the energy deposition in the magnetic field to the energy deposition in the absence of the field was evaluated and compared to the results of measurements. Each simulation was performed such as to achieve a statistical uncertainty of the simulation below $0.1 \%$.

\subsection{Polarity and recombination corrections}

Polarity and recombination correction factors were measured in an experimental setup similar to that of Section 2.2, except that a water phantom with a larger lateral width of $9.5 \mathrm{~cm}$ was used together with a field size of $10 \times 8 \mathrm{~cm}$. As this required a larger distance of the pole shoes, the magnetic field strength was limited to $0.35 \mathrm{~T}$ for these measurements. The effect on the chamber reading using potentials of opposite polarity was determined by the methods from the TRS-398 dosimetry protocol $[20]$, by

$k_{p o l}=\frac{\left|M_{+}\right|+\left|M_{-}\right|}{2 M}$

where $\mathrm{M}$ is the electrometer reading obtained with the polarity for the everyday use of the chamber which was $+300 \mathrm{~V}$ in our case, and $M_{+}=$ $+300 \mathrm{~V}$ and $M_{-}=-300 \mathrm{~V}$, respectively.

The two-voltage method was used to determine the ion recombination correction factor $k_{s}$ :

$k_{s}=a_{0}+a_{1}\left(\frac{M_{1}}{M_{2}}\right)+a_{2}\left(\frac{M_{1}}{M_{2}}\right)^{2}$

where $M_{1}$ is the normal operating voltage (300 V) and $M_{2}$ is a lower voltage $(100 \mathrm{~V})$, and the constants $a_{0}=1.198, a_{1}=-0.875$ and $a_{2}=$ 0.677 are taken from TRS-398 dosimetry protocol [20].

Measurements were carried out in transverse orientation (Fig. 3a), with a $0.35 \mathrm{~T}$ magnetic field in $+\mathrm{X}$ and $-\mathrm{X}$ direction as well as at $0 \mathrm{~T}$. The measurement was repeated three times to determine the uncertainty of $k_{p o l}$ and $k_{s}$.

\subsection{Ionization chamber response under axial rotation}

The experiments were performed with a similar setup as that in 


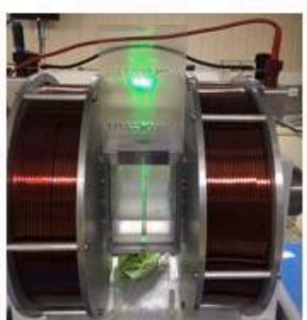

(a)

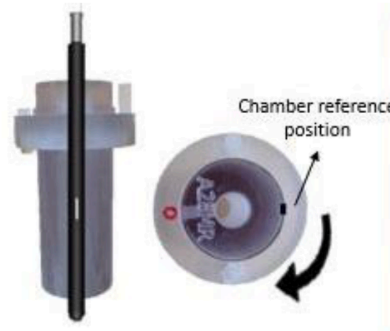

(b)

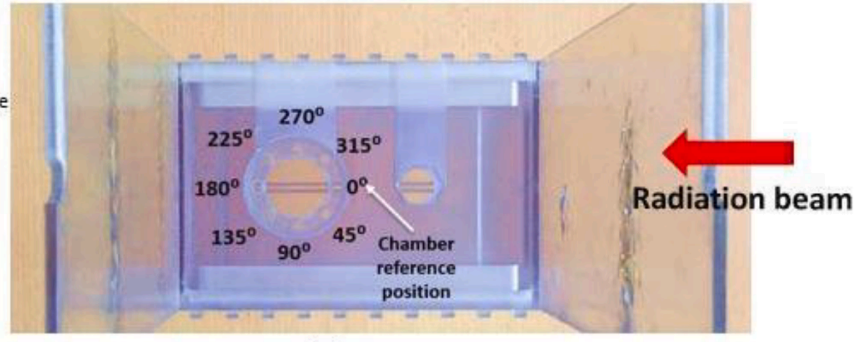

(c)

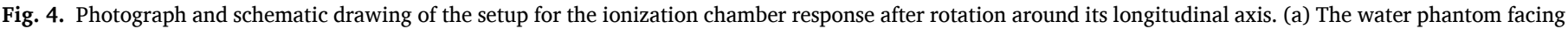

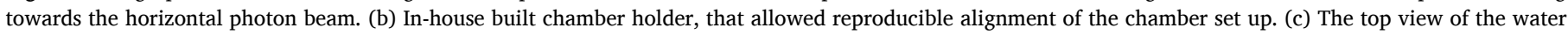
phantom, with insert for defined chamber rotation around its longitudinal axis.
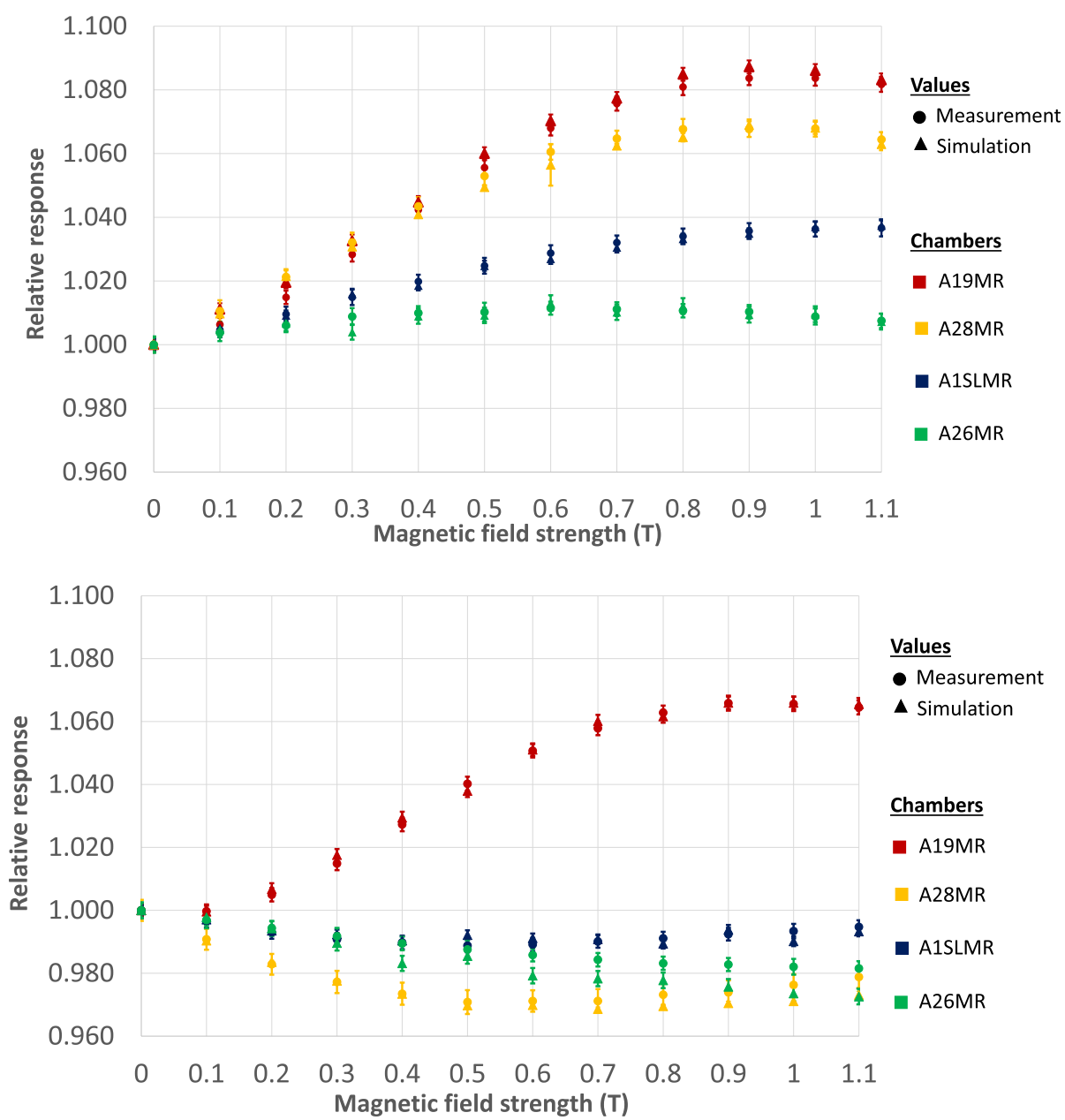

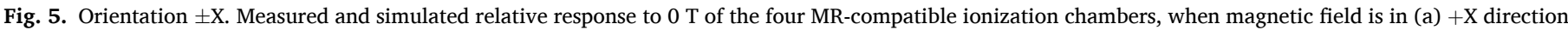
and (b) $-\mathrm{X}$ direction. The error bars on each data point show the relative uncertainty, which is below $1.0 \%$.

Section 2.3. The chamber position was fixed using an in-house built holder. The holder allowed chamber rotations to defined azimuth angles around its longitudinal axis, and one set of measurements was performed every 45 degrees (Fig. 4). Measurements were carried out in transverse orientation (Fig. 3a), at $0 \mathrm{~T}$ and with a magnetic field of 0.35 $\mathrm{T}$ in $+\mathrm{X}$ and $-\mathrm{X}$ direction.

\section{Results}

3.1. Relative response for different magnetic field strengths and orientations

Fig. 5 shows the measured and simulated response of all ionization chambers relative to the $0 \mathrm{~T}$ value as a function of the magnetic field strength for orientation $\pm \mathrm{X}$ (Fig. 3a). The measurement shows very good agreement with the simulation.

When the magnetic field is oriented in $+\mathrm{X}$ direction, the A19MR Farmertype chamber response increases gradually to a maximum of 

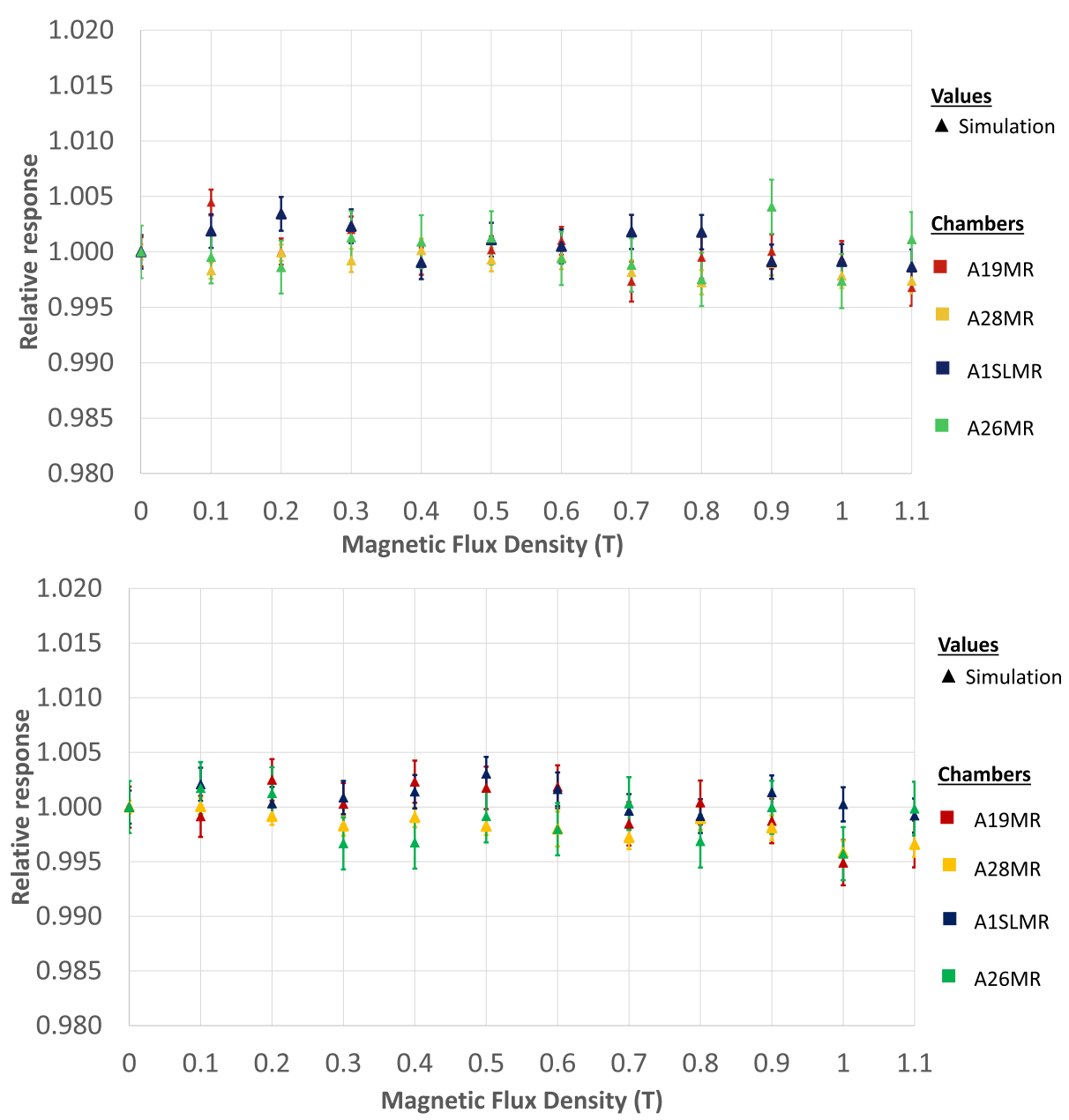

Fig. 6. Orientation $\pm \mathrm{Y}$. Simulated response of the four MR-compatible ionization chambers, when the magnetic field is in (a) $+\mathrm{Y}$ direction and (b) $-\mathrm{Y}$ direction, relative to the value at $0 \mathrm{~T}$. The error bars on each data point show the relative uncertainty, which is below $1.0 \%$.

$+8.5 \%$ at $0.9 \mathrm{~T}$ before decreasing again at higher magnetic field strengths. For A28MR, A1SLMR and A26MR, the response gradually increases with the magnetic field strength to $+6.8 \%$ at $0.9 \mathrm{~T},+3.7 \%$ at $1.1 \mathrm{~T}$ and $+1.6 \%$ at $0.6 \mathrm{~T}$ respectively. By reversing the magnetic field direction (magnetic field in $-\mathrm{X}$ direction), A19MR yields a maximum increase of $+6.5 \%$ at $0.9 \mathrm{~T}$ while the response of A28MR, A1SLMR and A26MR shows the opposite trend: their curves decrease with increasing magnetic field strength to a minimum value of $-3.0 \%$ at $0.6 \mathrm{~T},-1.2 \%$ at $0.5 \mathrm{~T}$ and around $-2.5 \%$ at $1.1 \mathrm{~T}$, respectively.

For the A26MR chamber, a difference between the simulated and the measured chamber response is observed, which is about $1 \%$ at a magnetic field strength of $0.6 \mathrm{~T}$.

The simulation results for a magnetic field parallel to the chamber in orientation $\pm \mathrm{Y}$ (Fig. 3b) are shown in Fig. 6. A systematic alteration in chamber response due to the presence of the magnetic field could not be found, irrespective of the field orientation. The response alteration shows only minor variations between 1.004 and 0.996 for both polarities, which is much smaller than the statistical uncertainty of the simulation amounting up to $0.24 \%$.

For the magnetic field parallel to the beam in orientation $\pm \mathrm{Z}$ (Fig. 3c), no pronounced dependence of the field orientation on the chamber response could be found (Fig. 7). The simulation yielded relative response values within $1 \%$ for all four chambers and in both magnetic field directions.

\subsection{Polarity and recombination corrections}

The corrections for the polarity effect and ion recombination effect for all four chambers with and without a magnetic field in transverse orientation are presented in Table 2. No significant differences were found at magnetic field strengths of $0.35 \mathrm{~T}$ in neither orientation of the magnetic field.

\subsection{Ionization chamber response under axial rotation}

Fig. 8 shows the relative chamber response when the ionization chambers were rotated about their longitudinal axis, with and without a magnetic field. For the chambers A28MR, A1SLMR and A26MR, no effect was observed. However, an angular dependence of the response was observed for A19MR farmer-type chamber independent of the presence of the magnetic field.

\section{Discussion}

\subsection{Relative response for different magnetic field strengths and orientations}

Four ionization chambers were investigated for their response alteration in presence of differently oriented magnetic fields. As the magnetic flux dentity was limited to $1.1 \mathrm{~T}$, due to the capabilities of the employed electromagnet, our study does not cover the full range of commercially available MR-linacs. Nevertheless, it adds valuable data at 

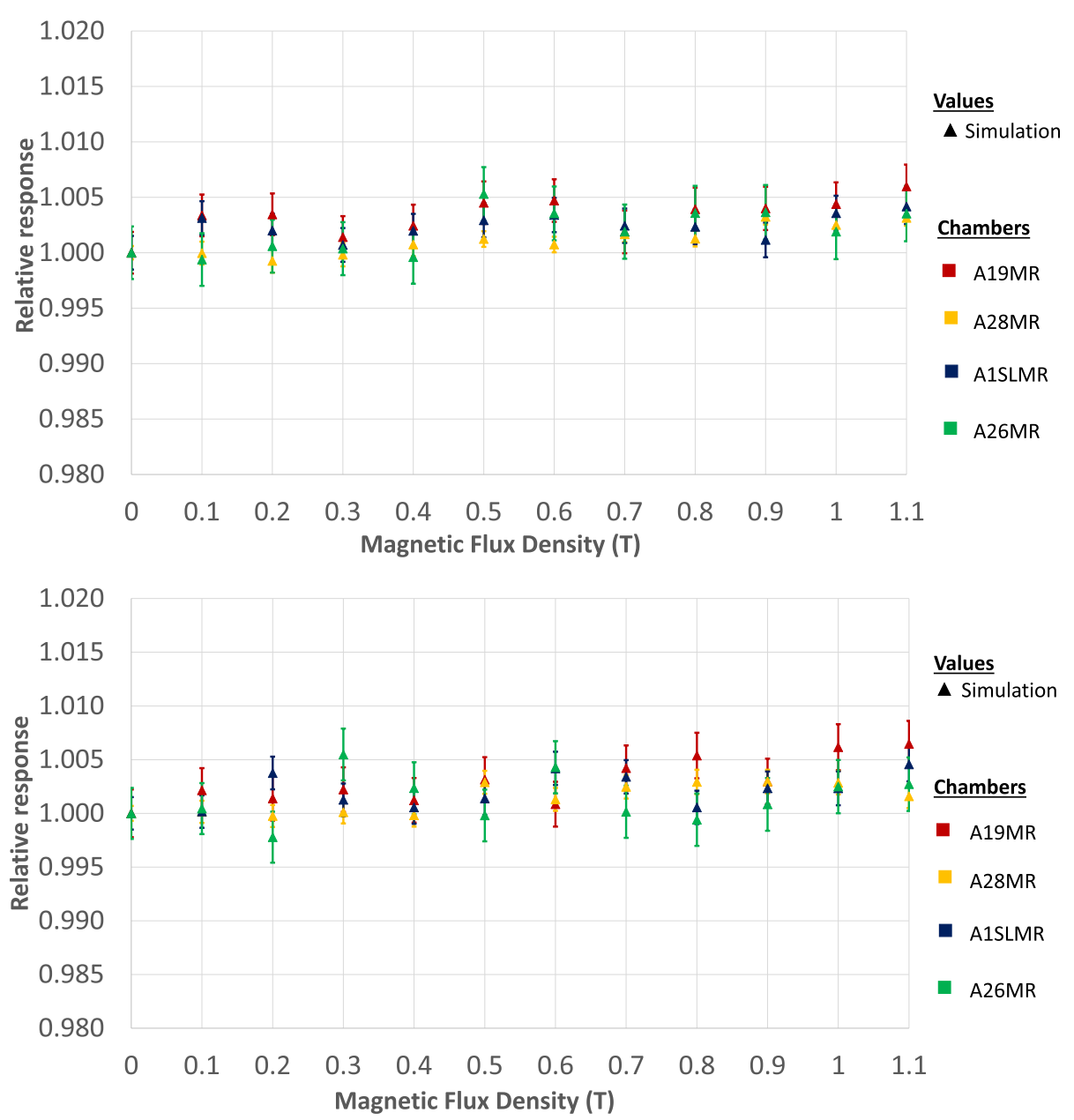

Fig. 7. Orientation $\pm Z$. Simulated response of four MR-compatible ionization chambers, when magnetic field is in (a) $+\mathrm{Z}$ direction and (b) $-\mathrm{Z}$ direction, relative to the value at $0 \mathrm{~T}$. The error bars on each data point show the relative uncertainty, which is below $1.0 \%$.

Table 2

Ratio of measured $\mathrm{k}_{\mathrm{pol}}$ and $\mathrm{k}_{\mathrm{s}}$ in the presence of magnetic field of $0.35 \mathrm{~T}$ to the value measured without magnetic field.

\begin{tabular}{|c|c|c|c|c|c|c|c|c|}
\hline \multirow[b]{2}{*}{ Magnetic field direction } & \multicolumn{2}{|c|}{ A19MR } & \multicolumn{2}{|c|}{ A28MR } & \multicolumn{2}{|c|}{ A1SLMR } & \multicolumn{2}{|c|}{ A26MR } \\
\hline & $+\mathrm{X}$ & $-\mathrm{X}$ & $+\mathrm{X}$ & $-\mathrm{X}$ & $+\mathrm{X}$ & $-\mathrm{X}$ & $+\mathrm{X}$ & $-\mathrm{X}$ \\
\hline $\mathrm{k}_{\mathrm{pol}}(0.35 \mathrm{~T}) / \mathrm{k}_{\mathrm{pol}}(0 \mathrm{~T})$ & 1.000 & 0.999 & 1.001 & 1.000 & 1.001 & 0.999 & 1.000 & 0.997 \\
\hline $\mathrm{k}_{\mathrm{s}}(0.35 \mathrm{~T}) / \mathrm{k}_{\mathrm{s}}(0 \mathrm{~T})$ & 1.000 & 1.000 & 1.000 & 1.001 & 1.000 & 1.000 & 0.999 & 0.999 \\
\hline
\end{tabular}

lower field strengths and the performed benchmarking of the Monte Carlo simulations allows in principle to extrapolate the data up to $1.5 \mathrm{~T}$. While the results of our study are in general inline those of other studies, our study adds important data for several ionization chambers dedicated for use in MR-linacs.

When the magnetic field was directed towards the $+\mathrm{X}$ direction, an increase of the chamber response in the presence of a magnetic field was seen. For the A19MR Farmer type chamber, the chamber response also increased when the magnetic field direction was reversed, while all other chambers exhibited a decreased response. The behavior of different Farmer type chambers in magnetic field has been described in Spindeldreier et al. [12], where it was shown that the transverse magnetic field leads to a stronger dose deposition at the tip $(+\mathrm{X})$ or at the stem $(-X)$ of the chamber depending on the orientation, due to Lorentz force deflection of the secondary electrons. The maximum observed dose response in this study in the $+\mathrm{X}$ magnetic field direction was 1.085, $1.068,1.037$ and 1.016 for the chambers A19MR, A28MR, A1SLMR and A26MR, respectively. This effect can be related to the different sensitive volume of the ionization chambers. The path length of secondary electrons in the cavity gets larger in magnetic fields as a result of its circular path. Meijsing et al. [9] illustrated the initial increase of the average path length, as the electron trajectories are bent in the direction of the longitudinal axis of the ionization chamber. For a given magnetic field strength, the mean electron path length in a chamber with larger volume will be larger than in the case of smaller chambers. Depending on the energy of the electrons for a certain magnetic field strength, the path length will start to decrease. In contrast, when the magnetic field is directed towards the $-\mathrm{X}$ direction, the response of the A19MR chamber remained below the response for the other polarity. As for the other three chambers, an opposite trend was observed; the reason could be the geometry of the ionization chamber (length of the collector and the much smaller sensitive volume) (Table 1 ).

The simulation results of transverse $( \pm X)$ orientation are in good agreement with the experimental measurements for A19MR, A28MR and A1SLMR ionization chambers. For the A26MR ionization chamber, the simulated chamber response differs by about $1 \%$ from the measured response for a magnetic flux density above $0.6 \mathrm{~T}$ oriented to the negative direction, where simulated values are lower than the experimental 

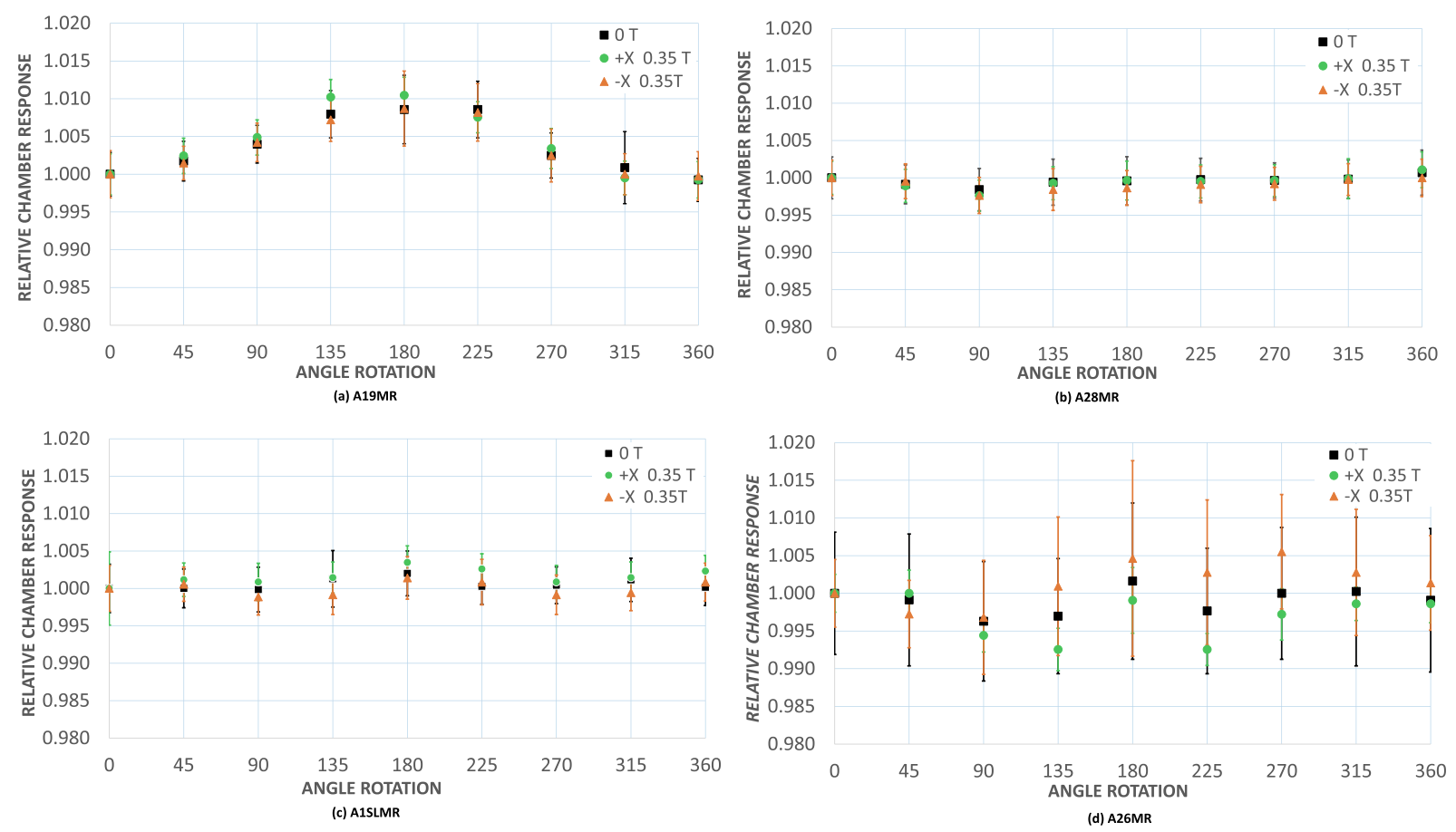

Fig. 8. Relative chamber response as a function of axial rotation angle in steps of $45^{\circ}$. The measurements are normalized to the value at 0 .

measurements. This deviation might be caused by two reasons: (i) inaccurate geometric modeling of small details in the chamber model, specifically small air regions close to the stem, and (ii) pertubations of the electric field near to the guard electrode, which locally changes the charge collection efficiency and leads to dead volumes. These pertubations have been investigated in detail by Pojtinger et al. by simulating the electric field by finite element methods and considering the resulting dead volume resulted in a better agreement between measured and simulated data [19]. Due to the small volume of only $0.05 \mathrm{~cm}^{3}$, the response of the A26MR chamber was found to be very sensitive to small geometric changes in the chamber model and the same sensitivity can be expected for the dead volume effect.

For the other three chambers, simulation and measurements agreed better and no adjustment of the dead volume in the ionization chamber model was necessary. Small changes up to $0.5 \%$ for both orientations of the magnetic fields are expected in $\pm \mathrm{Y}$, where the electrons are deflected laterally. Similarly, the response shows an increase of $0.7 \%$ for magnetic fields in $\pm \mathrm{Z}$ orientation, where the electrons are focused along the magnetic field. No noticeable dependence on the chamber radius and the magnetic field orientation was observed for these field orientations. The error bars in the Figure [5-7] characterizes the measurement uncertainty (standard deviation of three measurements plus the uncertainty for temperature and pressure as well as the reading uncertainty). The simulation goal was, to achieve an acceptable level of statistical uncertainty (within 1\%) by the optimal combination of VRTs.

\subsection{Polarity and recombination corrections}

The characteristics of the ionization chambers, such as material and geometry, influence the chamber response by scattered electrons into the collecting volume. Recombination and polarity corrections could, in principle, be influenced by the magnetic field. However, as outlined by the ratio of polarity and recombination effects with and without magnetic field, no significant changes were found [8]. This finding, however, is limited to low magnetic field strengths of $0.35 \mathrm{~T}$. The transverse orientation of the magnetic field exhibited the largest differences in the field-strength-dependent response, thus it may also be expected that the polarity and recombination effects to be the largest in this orientation.

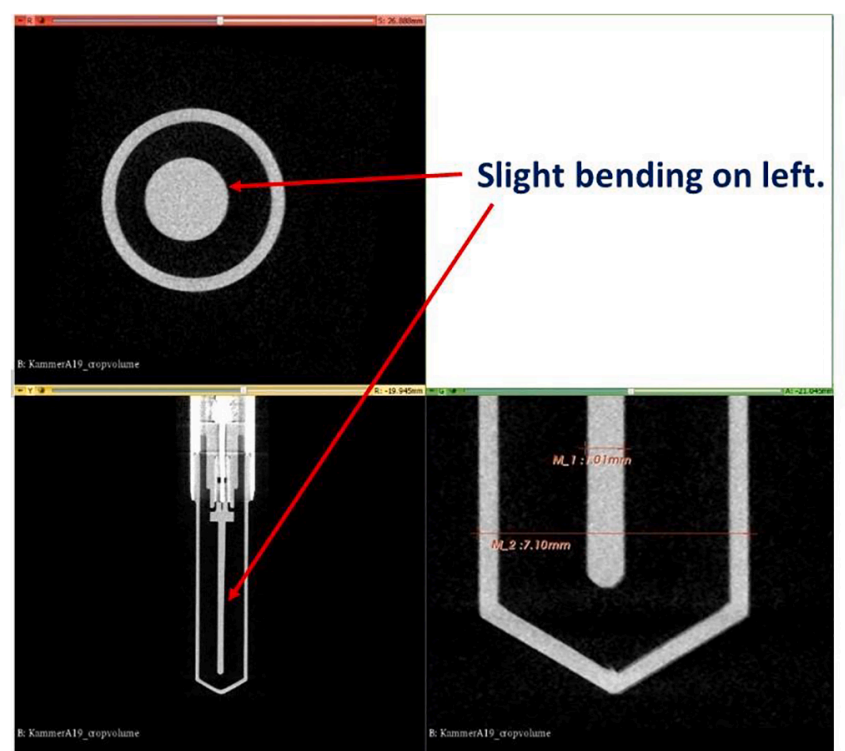

Fig. 9. Micro-CT of the A19MR Farmer-type chamber showing a slight bending of the central electrode.

However the effects are negligible in the transverse orientation, it can be expected that they are also negligible for other orientations of the magnetic field. Nevertheless, this finding could be different for other chamber types due to difference in material composition and designs.

\subsection{Ionization chamber response under axial rotation}

The chamber response of the chambers A28MR, A1SLMR and A26MR remained unaffected by axial rotations in a magnetic field of $0.35 \mathrm{~T}$. This may be explained by the fact that the chambers have an axial symmetry. As for the A19MR Farmer type chamber, a gradual increase of the chamber response with angle up to $180^{\circ}$ was observed. This was explained by a slight bending of the central electrode, which has 
been detected in a micro-CT image of the chamber (Fig. 9). This implies that the mechanical integrity of ionization chamber is important irrespective of the presence of a magnetic field.

\section{Conclusion}

The response of commercially available MR-compatible ionization chambers were examined as a function of the magnetic field strength and its orientation relative to the photon beam. In addition, polarity and ion recombination effects and the dependence of the response on axial rotations of the chamber were analyzed. With increasing sensitive volume of the chambers, the dependence of the response on the magnetic field strength increases as well for perpendicular orientations of magnetic field, chamber axis and beam. For the other two orientations, no appreciable change in chamber response was found in the presence of magnetic fields up to $1.1 \mathrm{~T}$. No pronounced changes of the polarity and recombination corrections were found in the presence of $0.35 \mathrm{~T}$ magnetic field. The response of the chambers did not change under axial rotation of the chamber, independently of the presence of the magnetic field. The data presented here add comprehensive knowledge on the response of MR-compatible ionization chambers in magnetic fields.

\section{Declaration of Competing Interest}

The authors declare that they have no known competing financial interests or personal relationships that could have appeared to influence the work reported in this paper.

\section{References}

[1] Fallone B, Murray B, Rathee S, Stanescu T, Steciw S, Vidakovic S, Blosser E, Tymofichuk D. First $\mathrm{mr}$ images obtained during megavoltage photon irradiation from a prototype integrated linac-mr system. Med Phys 2009;36(6Part1):2084-8.

[2] Keall PJ, Barton M, Crozier S. The australian magnetic resonance imaging-linac program, in: Seminars in radiation oncology, vol. 24, Elsevier; 2014. p. 203-206.

[3] Dempsey J, Dionne B, Fitzsimmons J, Haghigat A, Li J, Low D, Mutic S, Palta J, Romeijn H, Sjoden G. We-e-vala-06: a real-time mri guided external beam radiotherapy delivery system. Med Phys 2006;33(6Part20). 2254-2254.

[4] Klüter S. Technical design and concept of a $0.35 \mathrm{t}$ mr-linac. Clin Transl Radiat Oncol 2019;18:98-101.
[5] Lagendijk JJ, Raaymakers BW, Raaijmakers AJ, Overweg J, Brown KJ, Kerkhof EM, van der Put RW, Hårdemark B, van Vulpen M, van der Heide UA. Mri/linac integration. Radiother Oncol 2008;86(1):25-9.

[6] Raaijmakers A, Raaymakers BW, van der Meer S, Lagendijk JJ. Integrating a mri scanner with a $6 \mathrm{mv}$ radiotherapy accelerator: impact of the surface orientation on the entrance and exit dose due to the transverse magnetic field. Phys Med Biol 2007;52(4):929.

[7] Raaijmakers A, Raaymakers BW, Lagendijk JJ. Integrating a mri scanner with a 6 mv radiotherapy accelerator: dose increase at tissue-air interfaces in a lateral magnetic field due to returning electrons. Phys Med Biol 2005;50(7):1363.

[8] Smit K, Van Asselen B, Kok J, Aalbers A, Lagendijk J, Raaymakers B. Towards reference dosimetry for the mr-linac: magnetic field correction of the ionization chamber reading. Phys Med Biol 2013;58(17):5945.

[9] Meijsing I, Raaymakers BW, Raaijmakers A, Kok J, Hogeweg L, Liu B, et al. Dosimetry for the mri accelerator: the impact of a magnetic field on the response of a farmer ne2571 ionization chamber. Phys Med Biol 2009;54(10):2993.

[10] Reynolds M, Fallone B, Rathee S. Dose response of selected ion chambers in applied homogeneous transverse and longitudinal magnetic fields. Med Phys 40 (4).

[11] O'brien D, Roberts D, Ibbott G, Sawakuchi G. Reference dosimetry in magnetic fields: formalism and ionization chamber correction factors. Med Phys 2016;43 (8Part1):4915-27.

[12] Spindeldreier CK, Schrenk O, Bakenecker A, Kawrakow I, Burigo L, Karger CP, et al. Radiation dosimetry in magnetic fields with farmer-type ionization chambers: determination of magnetic field correction factors for different magnetic field strengths and field orientations. Phys Med Biol 2017;62(16):6708. http://stacks. iop.org $/ 0031-9155 / 62 / i=16 / a=6708$.

[13] Jacco A, Ilias B, Leon ADP, Simon D, Ralf-Peter K, Christian K, Bram VA, Jochem W. Reference dosimetry in mri-linacs: evaluation of available protocols and data to establish a code of practice, Phys Med Biol.

[14] Standardimaging Inc. (USA), Exradin MR compatible ion chambeirs, https://www. standardimaging.com/exradin/thimble-ion-chambers (2016, assessed on 2018-1105).

[15] Kawrakow I, Mainegra-Hing E, Rogers DWO, Tessier F, Walters BRB. The EGSnrc code system: Monte Carlo simulation of electron and photon transport, Tech. Rep. PIRS-701, National Research Council Canada; 2017.

[16] Kawrakow I. Accurate condensed history Monte Carlo simulation of electron transport. I. EGSNRC, the new EGS4 version. Med Phys 2000;27(3):485-98.

[17] Wulff J, Zink K, Kawrakow I. Efficiency improvements for ion chamber calculations in high energy photon beams. Med Phys 2008;35(4):1328-36.

[18] Mohan R, Chui C, Lidofsky L. Energy and angular distributions of photons from medical linear accelerators. Med Phys 1985;12(5):592-7.

[19] Pojtinger S, Kapsch R-P, Dohm OS, Thorwarth D. A finite element method for the determination of the relative response of ionization chambers in mr-linacs: simulation and experimental validation up to 1.5 t. Phys Med Biol 2019;64(13): 135011.

[20] Implementation of the International Code of Practice on Dosimetry in Radiotherapy (TRS 398): Review of Testing Results, No. 1455 in TECDOC Series (CD-ROM). International Atomic Energy Agency, Vienna; 2010. 